University of New Hampshire

University of New Hampshire Scholars' Repository

Space Science Center

Institute for the Study of Earth, Oceans, and

Space (EOS)

$5-29-2013$

\title{
An imaging neutron/gamma-ray spectrometer
}

Amanda C. Madden

University of New Hampshire - Main Campus

Peter F. Bloser

University of New Hampshire, Peter.Bloser@unh.edu

Dominique Fourguette

Michigan Aerospace Corp.

Liane Larocque

Michigan Aerospace Corp.

Matt Lewis

Michigan Aerospace Corp.

See next page for additional authors

Follow this and additional works at: https://scholars.unh.edu/ssc

Part of the Astrophysics and Astronomy Commons

\section{Recommended Citation}

Amanda C. Madden ; Peter F. Bloser ; Dominique Fourguette ; Liane Larocque ; Jason S. Legere ; Matt Lewis ; Mark L. McConnell ; Marissa Rousseau ; James M. Ryan; An imaging neutron/gamma-ray spectrometer . Proc. SPIE 8710, Chemical, Biological, Radiological, Nuclear, and Explosives (CBRNE) Sensing XIV, 87101L (May 29, 2013); doi:10.1117/12.2018075.

This Conference Proceeding is brought to you for free and open access by the Institute for the Study of Earth, Oceans, and Space (EOS) at University of New Hampshire Scholars' Repository. It has been accepted for inclusion in Space Science Center by an authorized administrator of University of New Hampshire Scholars' Repository. For more information, please contact Scholarly.Communication@unh.edu. 


\section{Authors}

Amanda C. Madden, Peter F. Bloser, Dominique Fourguette, Liane Larocque, Matt Lewis, Jason S. Legere, Mark L. McConnell, Marissa Rouseau, and James M. Ryan 


\title{
An imaging neutron/gamma-ray spectrometer
}

\author{
Amanda C. Madden ${ }^{a}$, Peter F. Bloser ${ }^{a}$, Dominique Fourgette ${ }^{b}$ Liane Larocque $^{b}$, \\ Jason S. Legere ${ }^{a}$, Matt Lewis ${ }^{b}$, Mark L. McConnell ${ }^{a}$, Marissa Rousseau ${ }^{a}$, James M. Ryan ${ }^{a}$ \\ ${ }^{a}$ Space Science Center, University of New Hampshire, 8 College Rd., Durham, NH USA 03824; \\ ${ }^{b}$ Michigan Aerospace Corp., 1777 Highland Drive, Suite B, Ann Arbor, MI USA 48108
}

\begin{abstract}
We present the test results of a neutron/gamma-ray imaging spectrometer for the identification and location of radioactive and special nuclear materials. Radioactive materials that could be fashioned into a radiation dispersal device typically emit gamma rays, while fissile materials such as uranium and plutonium emit both neutrons and gamma rays via spontaneous or induced fission. The simultaneous detection of neutrons and gamma rays is a clear indication of the presence of fissile material. The instrument works as a double-scatter telescope, requiring a neutron or gamma ray to undergo an interaction in two detectors to be considered a valid event. While this requirement reduces the detector efficiency, it yields information about the direction and energy of the incident particle, which is then used to reconstruct an image of the emitting source. Because of this imaging capability background events can be rejected, decreasing the number of events required for high confidence detection and thereby greatly improving its sensitivity. The instrument is optimized for the detection of neutrons with energies from 1-20 MeV and gamma rays from 0.4 to $10 \mathrm{MeV}$. Images and energy spectra for neutron and gamma rays are reported for several sources including depleted uranium and plutonium. In addition, the effect of neutron source shielding is investigated.
\end{abstract}

Keywords: neutrons, gamma rays, imaging, spectroscopy, standoff detection, Special Nuclear Materials, homeland security, radiation

\section{INTRODUCTION}

Radioactive and fissile materials are commonly used in the industrial, medical and power industries. With many sources available, the threat of a source going missing increases. These "orphaned" sources could potentially be fashioned into a Radiation Dispersal Device (RDD) or a nuclear weapon. The ability to remotely locate, measure and identify these sources is a serious concern for homeland security purposes. The Neutron Spectroscope (NSPECT) instrument developed by Michigan Aerospace Corp. and the University of New Hampshire aims to aid in this effort.

Because neutrons and gamma rays are neutral particles, they do not readily interact with material. Several methods can be used to increase the probability of a neutron detection, such as using a moderator or a large bulk detector. However, a moderator erases energy and directional information, and a bulk detector yields no directional information. For this reason, the NSPECT instrument utilizes the double-scatter telescope technique. While the double scatter requirement decreases the detector efficiency, it constrains of the trajectory of the incident neutron while also providing a measurement of the neutron energy. The ability to constrain the trajectory of a neutron or $\gamma$ ray allows for significant background rejection, improving detector sensitivity.

\section{THE DOUBLE SCATTER TECHNIQUE}

\subsection{Neutron Detection}

Neutrons in the $\mathrm{MeV}$ range, have large cross sections for elastic scattering. The simplistic kinematics involved in the two body problem of a neutron-proton interaction makes hydrogen a good choice for an interaction target. Organic scintillators typically have a hydrogen to carbon ratio of 1.0 to 2.0 , which varies with scintillator type. The large quantities of hydrogen present in organic scintillators makes this an ideal detector material for neutrons. To perform imaging, a single neutron with total energy $E_{n_{o}}$ must undergo an interaction in each of two scintillators in detector layers, D1 and D2. The neutron will transfer some portion of its energy $\left(\mathrm{E}_{p_{1}}\right)$ to a

Chemical, Biological, Radiological, Nuclear, and Explosives (CBRNE) Sensing XIV,

edited by Augustus Way Fountain, Proc. of SPIE Vol. 8710, 87101L · C) 2013

SPIE - CCC code: 0277-786X/13/\$18 - doi: 10.1117/12.2018075

Proc. of SPIE Vol. $871087101 \mathrm{~L}-1$ 
proton in a D1 scintillator, before continuing on to interact with with a scintillator in D2. The remaining energy of the scattered neutron can be determined from the time of flight (ToF), or how long it takes the neutron to travel from the first to the second detector. The scatter vector $\vec{n}_{s}$ is determined by the position of the D1 and D2 cells involved. The angle $\phi$ between the scattered neutron vector and the initial neturon vector $\vec{n}_{o}$ is calculated from kinematics: $\sin ^{2} \phi=E_{p 1} / E_{n_{o}}$. Using this, we can constrain the incident neutron trajectory to lie on the mantle of a cone of half angle $\phi$, centered around $\vec{n}_{s}$. By extending this cone out to an image plane, an event circle or ellipse is formed for each event. The intersection of many event circles in effect creates the neutron image of a source.

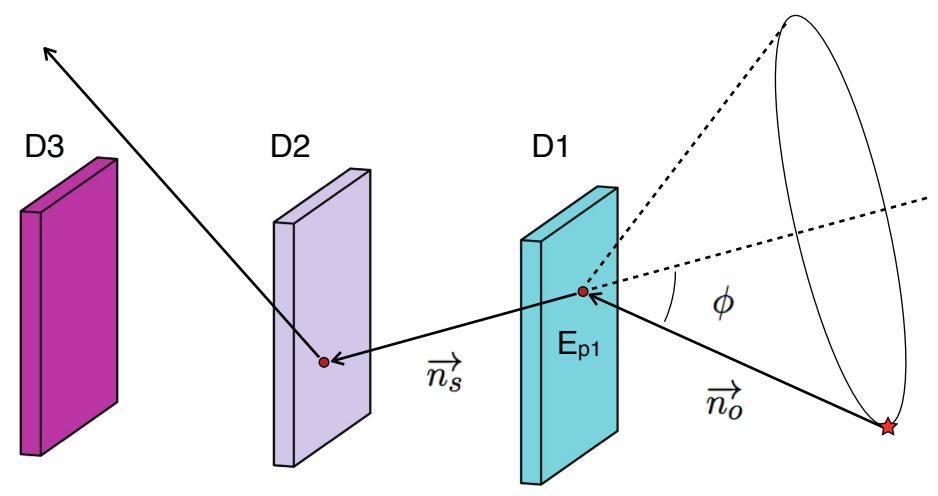

Figure 1. Neutron double-scatter kinematics.

\subsection{Gamma-ray Detection}

While the neutron analysis employs kinematic relationships to determine the energy and trajectory of the incident neutron, the process that governs the interaction of gamma-rays with scintillating material is Compton scattering. Again, we require a gamma ray with energy $\mathrm{E}_{\gamma_{o}}$ to interact in each of two detector layers. The gamma ray will deposit some fraction of its energy $\mathrm{E}_{1}$ in the first detector and continue on to the second layer, where it is fully absorbed, depositing the remainder of its energy $\mathrm{E}_{2}$. From the Compton equation, the angle between the scattered gamma ray $\overrightarrow{\gamma_{s}}$ and the incident gamma ray $\overrightarrow{\gamma_{o}}$ is $\cos \phi=1-m_{e} c^{2}\left(E_{2}^{-1}-E_{\gamma_{o}}^{-1}\right)$, provided that the $\gamma$ ray is fully absorbed. A ToF measurment provides no new information for this type of detection, because all gamma rays travel at the speed of light. However, this measurement can be used to differentiate between gamma rays and neutrons. The Compton telescope has seen extensive use in space for astrophysics research, in particular the COMPTEL experiment on the Compton Gamma Ray Observatory. ${ }^{1}$

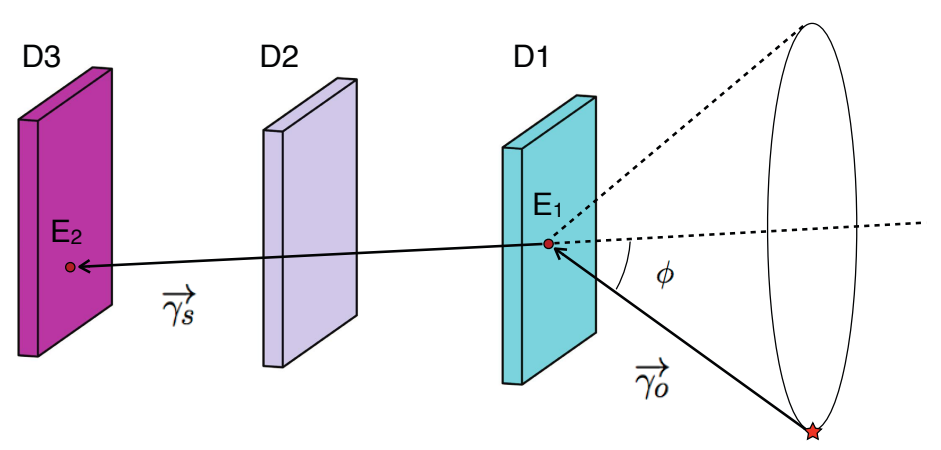

Figure 2. Compton scatter diagram. 


\section{NSPECT}

\subsection{The Dual-Species Detector Concept}

For the most effective neutron detection, a scintillator with a high hydrogen content as well as the ability to perform pulse shape discrimination (PSD) is required. In addition to the ToF, PSD helps distinguish between neutron and gamma events; an important requirement for environments with a large $\gamma$ background. Previous generations of neutron and gamma imaging telescopes have been constructed with either plastic or liquid scintillator in the forward layers. Liquid scintillators have the advantage of high hydrogen content and relatively good light output and PSD properties. However, liquid scintillating material is toxic and requires a more complex mechanical design for proper packaging and safety in transport and operation. For NSPECT to be a ruggedized, field ready instrument, plastic scintillators were used.

For effective $\gamma$ detection, a low density scattering material, such as an orgainic scintillator, and a high density calorimetric material for the second detector are implemented. All detection materials must be fast, in order to have an accurate ToF measurement for the neutrons and to increase the background rejection rate of $\gamma$-rays.

The partially populated instrument is shown in Figure 3. This three-layer system implements 1" cyllindrical scintillators, each with its own PMT and electronics read out representing independent data channels. Each layer is populated with a different type of scintillator. The first layer functions as a scatterer for both neutrons and $\gamma$ rays, and is made of low-Z plastic scintillator. The second layer is comprised of stilbene scintillators, which have PSD properties and is thus optimized for detecting the neutron scattered from a plastic D1 cell. The third layer is made of dense bismuth germanate (BGO), which is optimized for a $\gamma$ interaction.

Each layer is separated by a nominal distance of $37 \mathrm{~cm}$, but can be adjusted. This distance, along with corrections due to oblique trajectories, electronic walk and pulse height dependence is used to determine the ToF for the incident radiation. A $\gamma$-ray traversing this distance will take $1.25 \mathrm{~ns}$, while a neutron that leaves the D1 interaction site with $1 \mathrm{MeV}$ of energy takes about $30 \mathrm{~ns}$ to traverse the same distance. This difference in timing, in addition to the PSD properties, allows for the determination of radiation type.

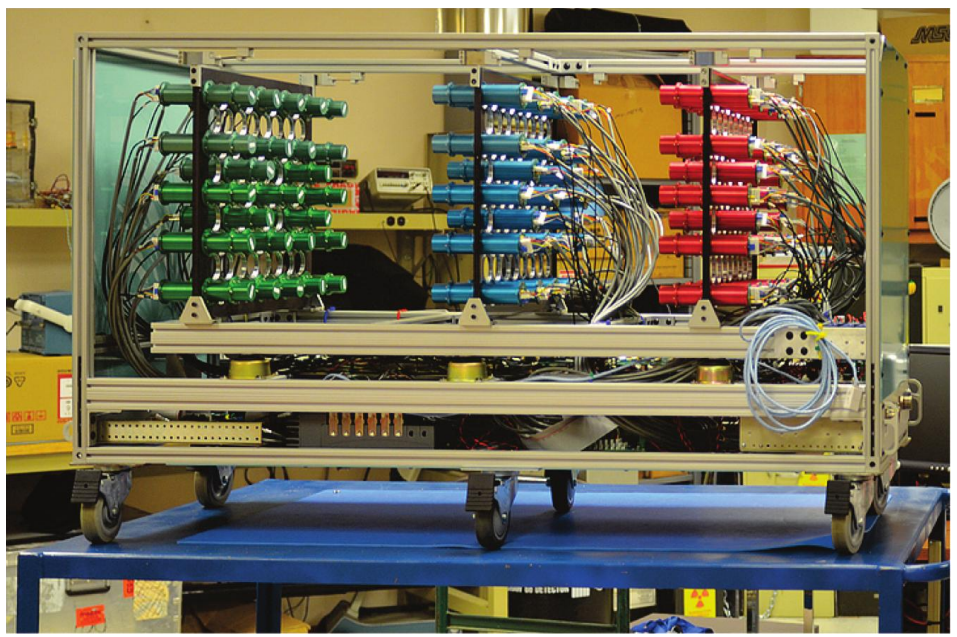

Figure 3. The NSPECT instrument. D1 is at the left.

\subsection{The NSPECT System}

The full instrument is designed to operate eight hours on battery power, with each detector layer populated with seventy-seven detector cells. Analog data is processed in an on-board computer, then digitized and formatted for transmission to a remote computer for real-time monitoring, and/or data archiving. The connection between the on-board and remote computers can be wireless or by ethernet cable. The instrument is controlled through the remote computer, nominally a laptop running LabVIEW ${ }^{\mathrm{TM}}$. 
After exposing the instrument to a source, double scatters in each data run are sorted using hardware coincidence criteria. For example, coincidences between D1 and D2 are assumed to be neutron events, where as coincidences between D1 and D3 or D2 and D3 are assumed to be $\gamma$ events. Furthermore, signals from D1 and D2 must occur within $50 \mathrm{~ns}$ of each other to be considered a coincident event, and the pulse height thresholds were set at $50 \mathrm{keV}_{\mathrm{ee}}$.

All cells were gain matched via high voltage adjustments, but finer scale gain matching takes place using calibration sources and is handled in the data processing. Also handled in the data processing is the non-linear pulse height response of organic scintillators, ToF corrections and the determination of radiation type from PSD data.

\subsection{Instrument Characterization}

\subsubsection{Fundamental parameters}

The accuracy of the ToF measurement directly contributes to the accuracy of the energy measurement of the neutron. To determine this accuracy, a ${ }^{60}$ Co source was centered between the D1 and D2 layers. This source emits quasi-simultaneous $\gamma$-rays, and thus the ToF measured should be $0 \mathrm{~s}$. To determine the width of this distribution, corrections for walk, first-dynode transit-time, cable length and geometrical separation were made. In order to exclude electronic noise and random coincidence, only events in a subset of the Compton continuum (200-800 keV) were analyzed. The resulting resolution was about 590 ps FWHM.

The $\gamma$-ray energy resolution is $20 \%$ at $662 \mathrm{keV}$, determined mostly from the resolution of the D3 BGO layer. Because no monochromatic neutron source was available, we estimate (from the D1 energy resolution and the ToF resolution) a neutron energy resolution of $10 \%$. The neutron energy threshold of the NSPECT system is $\sim 500 \mathrm{keV}_{\mathrm{pe}}$. This threshold is driven by the light output of both D1 and D2 and their associated front end electronics. The $\gamma$ energy threshold is $\sim 100 \mathrm{keV}_{\text {ee }}$.

\subsubsection{Imaging and spectral capabilities}

The factors that determine the ultimate imaging quality are the accuracy and precision of the scatter-angle calculation and the spatial-location uncertainty in the scattering sites. Since detectors are finite in size there is an uncertainty in the exact location of the interaction of the radiation with the scintillator. This uncertainty, in addition to effects from the detector's energy and ToF resolution, effectively "broaden" the event circle from a thin line to a line with finite thickness corresponding to these uncertainties. The net effect is quantified in the so-called angular resolution measure (ARM) distribution. The ARM quantity is the difference between the kinematically measured scattering angle (from energy and ToF) and the geometric scattering angle (known from the true source position). The width of the ARM distribution reflects the net uncertainties produced by the spatial, energy and ToF uncertainties, and is approximately $10^{\circ} \mathrm{FWHM}$ for both $\gamma$-rays and neutrons.

When a source location is known or identified in the constructed image, the ARM can be used to create a more accurate neutron and $\gamma$ spectrum. By selecting on events that pass within $12^{\circ}$ of the source location (roughly equal to the instrument's angular resolution) background events are removed from the spectrum, showing neutron and $\gamma$ characteristics more clearly.

\section{PERFORMANCE OF THE PARTIALLY POPULATED INSTRUMENT}

The binning of the following images was set so that one bin is half the angular resolution of the NSPECT system, i.e. $6^{\circ}$. A black circle indicating the approximate angular resolution of the instrument is overlayed on the images to show the known source location and to provide reference for the precision of the imaging technique. Many of these results use the same data as reported in $2012^{?}$, but with improved data analysis techniques. We also present data from two additional sources: depleted uranium and plutonium. 


\subsection{Neutron Imaging and Spectroscopy}

At a distance of $3 \mathrm{~m}$ from D1, we placed a ${ }^{252} \mathrm{Cf}$ source on the instrument optical axis. A distance of $3 \mathrm{~m}$ is enough to avoid significant parallax issues. We then moved the source $1 \mathrm{~m}$ orthogonally off axis (maintaining the same normal distance to the D1 plane.) The resulting images from these two exposures are shown in Figure 4a. and b. The count spectrum from both exposures is shown in Figure 4 c. and has the Watt spectrum superposed. ${ }^{2}$ The instrument threshold in this configuration is about $1 \mathrm{MeV}$, catching the majority of the Watt spectrum. In addition, the resemblance of the count spectrum to the neutron intensity spectrum implies that the instrument response is largely diagonal and varies little from $1 \mathrm{MeV}$ to $10 \mathrm{MeV}$. The diagonal nature of the response function means that the energy of a registered neutron is roughly that of the true neutron (with attendant broadening) with no skewness in the form of a "Compton-like" tail. We also note that the shape of the count spectrum varies little from on-axis to $18^{\circ}$ off axis.

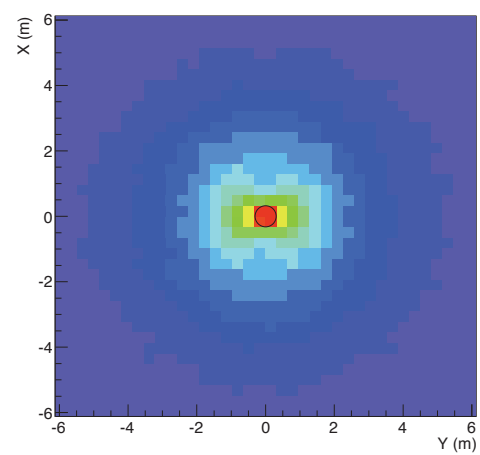

4a. The neutron image of an on-axis ${ }^{252} \mathrm{Cf}$ source.

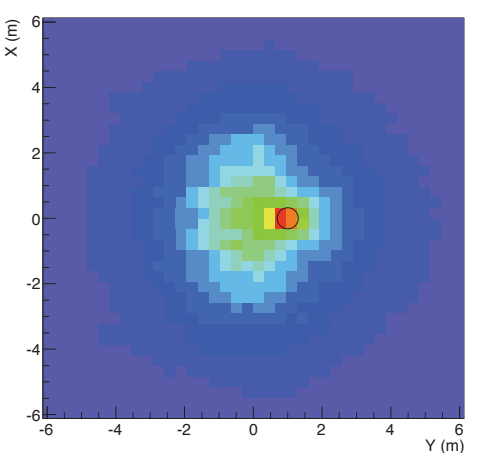

4b. The neutron image of a ${ }^{252} \mathrm{Cf}$ source $1 \mathrm{~m}$ off axis.

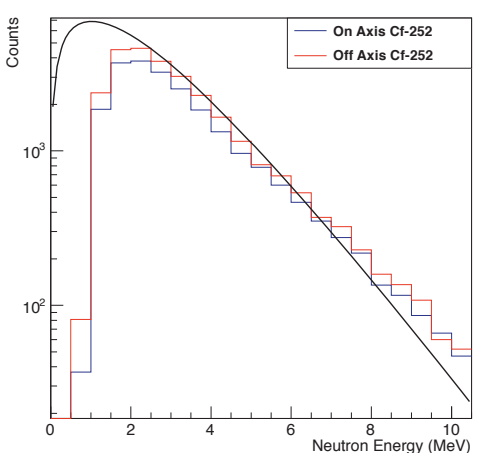

4c. On and off axis ${ }^{252} \mathrm{Cf}$ source count spectra.

Figure 4. On and off axis ${ }^{252} \mathrm{Cf}$ sources.

\section{$4.2 \gamma$-ray Imaging and Spectroscopy}

We performed a similar test with a ${ }^{137} \mathrm{Cs}$ source, again with the source on axis and $1 \mathrm{~m}$ off axis at a normal distance of $2 \mathrm{~m}$. The images from those exposures are shown in Figures 5a. and b. As with the neutrons, a spectrum was produced with a $12^{\circ} \mathrm{ARM}$ cut (Figure 5c.) The peaks from the exposures of on and off axis agree in peak energy to within $1 \%$. The nature of the $\gamma$ source is evident when such high quality spectra are collected, even with BGO quality resolution. 


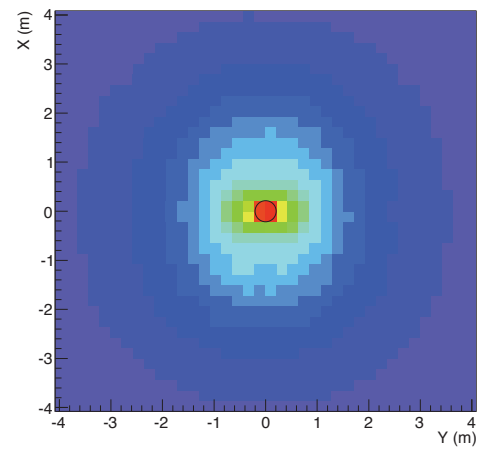

5a. The gamma image of an on-axis ${ }^{137} \mathrm{Cs}$ source.

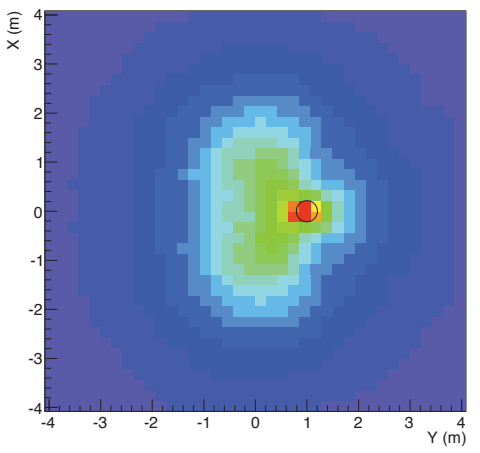

5b. The gamma image of a ${ }^{137} \mathrm{Cs}$ source $1 \mathrm{~m}$ off axis.

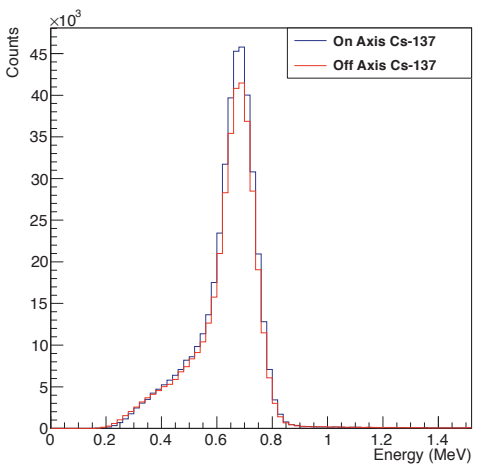

5c. On and off axis ${ }^{137} \mathrm{Cs}$ source count spectra.

Figure 5. On and off axis ${ }^{137} \mathrm{Cs}$ sources.

\subsection{Multiple Neutron Sources}

Next we tested the system's ability to recognize multiple neutron sources in the field of view. With only one ${ }^{252} \mathrm{Cf}$ source available, two separate exposures were taken- one $1 \mathrm{~m}$ off axis to the right and the other $0.85 \mathrm{~m}$ to the left. The two data files were then concatenated and processed as one. This process produces the same result except in the case that the two sources produce count rates too high for the electronics of the system. This was not the case here-the count rates from the individual runs were less than $6 \%$ of the maximum system intensity. Shown below (Fig. 6) is the dual-source image and the raw, unselected count spectrum. The exposure times for the runs were equal, but short compared to those of Section 4.1. We note that the exponential Watt spectrum emerges with no special data selections or processing. This implies that a many-fragmented quantity of fissile material with no fragment producing a coherent image would still be recognized, because of a clear Watt-like count spectrum.

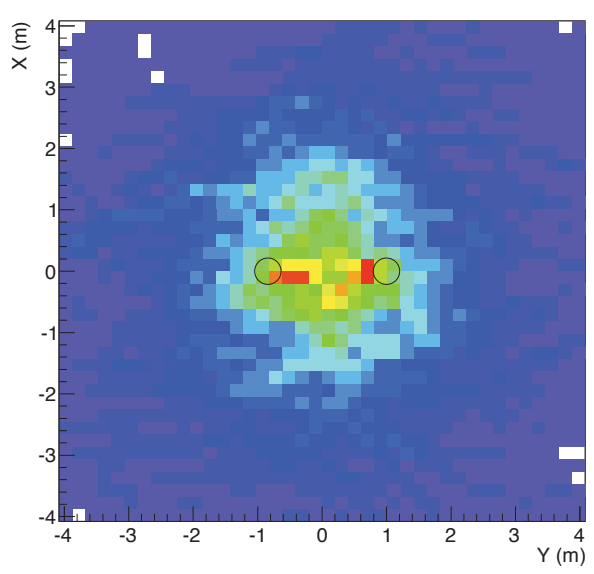

6a. The neutron image of two ${ }^{252} \mathrm{Cf}$ sources.

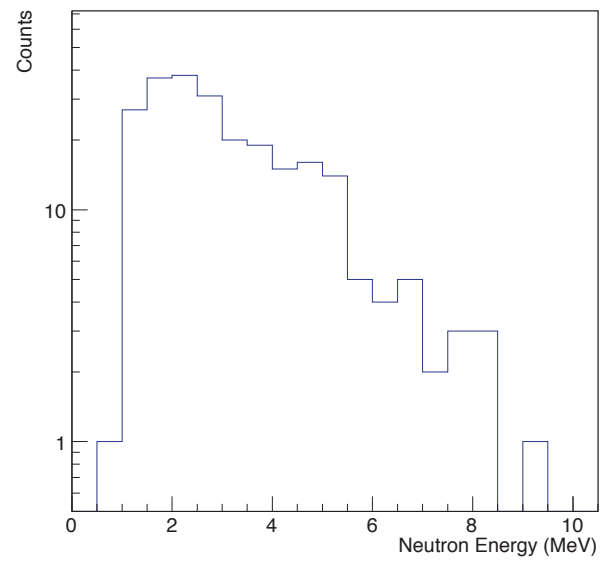

6b. The raw, unselected neutron count spectrum from the exposures in Figure 6a.

Figure 6. Multiple ${ }^{252} \mathrm{Cf}$ sources. 


\subsection{Gamma Interference in Neutron Detection}

A potentially confounding situation is that of a neutron source in an intense $\gamma$ field. This is the problem that drives the requirement for a neutron detector to have a gamma rejection ratio exceeding $10^{6}$. To test this, we put the ${ }^{137} \mathrm{Cs}$ source on axis with the ${ }^{252} \mathrm{Cf}$ sources $1 \mathrm{~m}$ off axis at a normal distance from the front panel of the instrument of $2 \mathrm{~m}$. Because of the intensity of the source and the high efficiency of NSPECT to detect $\gamma$ rays, the $\gamma$ signal is much greater than the neutron signal. The two sources were imaged and processed twice, first as a totally $\gamma$ source(s) and then as a neutron source(s). The neutron image and spectrum of Figure 7 clearly show the presence of a neutron source off axis, since the addition of a gamma emitting source should not affect the detection of neutrons. The result of the $\gamma$ imaging is shown in Figure 8. Both sources are present through the $\gamma$ filter, but because the $\gamma$ emission from the ${ }^{137} \mathrm{Cs}$ source is roughly 2.5 times the $\gamma$ emission from the ${ }^{252} \mathrm{Cf}$ source, it is the dominant presence in the figure.

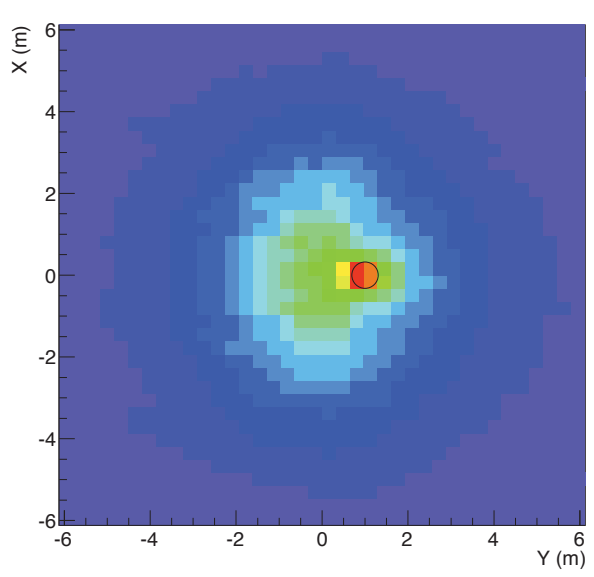

7a. The neutron image of a ${ }^{137} \mathrm{Cs}$ source on axis and a ${ }^{252} \mathrm{Cf}$ source $1 \mathrm{~m}$ off axis.

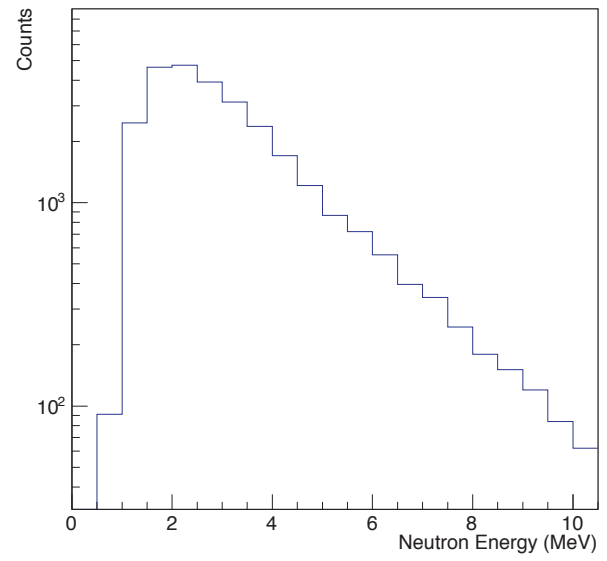

7b. The neutron spectrum from the region around the maximum neutron signal in Figure 7a.

Figure 7. Neutron imaging of a ${ }^{252} \mathrm{Cf}$ and ${ }^{137} \mathrm{Cs}$ source.

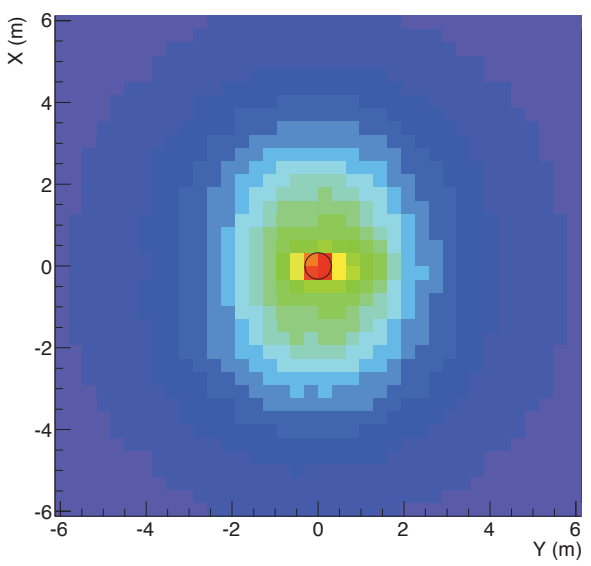

8a. The $\gamma$ image of a ${ }^{137} \mathrm{Cs}$ source on axis and a ${ }^{252} \mathrm{Cf}$ source $1 \mathrm{~m}$ off axis.

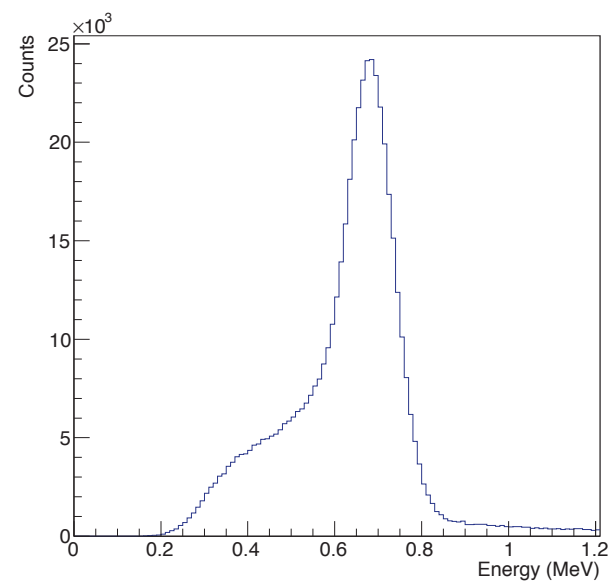

8b. The $\gamma$ spectrum around the maximum gamma signal in Figure 8a.

Figure 8. Gamma imaging of a ${ }^{252} \mathrm{Cf}$ and ${ }^{137} \mathrm{Cs}$ source. 
We can be more critical by imposing energy filters on the $\gamma$ image in Fig. 8. The $\gamma$ data in a window around $662 \mathrm{keV}$, specifically 325 to $800 \mathrm{keV}$ is shown in Fig 9a. The image is hardly distinguishable from that in Fig. 8. It is clear from this image and spectrum that a ${ }^{137} \mathrm{Cs}$ source is present at the origin of the NSPECT coordinate system. Using this information we can reanalyze the $\gamma$ data by imaging only events in the complementary energy range to Figure 9a. This allows us to look at the gamma events originating from an additional source. The results of these restrictions on gamma ray events is shown in Figure 9b.

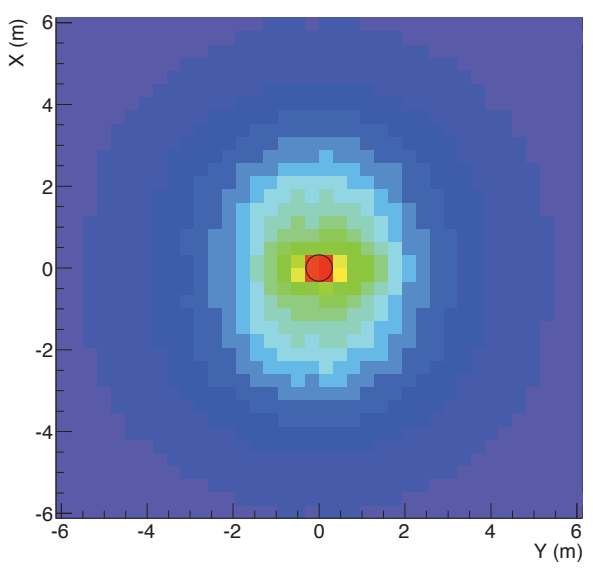

9a. The $\gamma$ image in an energy window around 662 $\mathrm{keV}$.

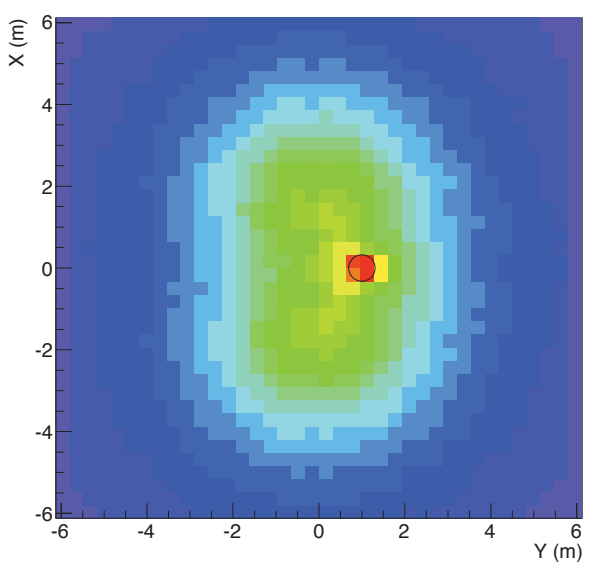

9b. The $\gamma$ image excluding the energy window around $662 \mathrm{keV}$

Figure 9. Gamma imaging of a ${ }^{252} \mathrm{Cf}$ and ${ }^{137} \mathrm{Cs}$ source with selections on energy.

\subsection{Shielded Sources}

One could expect under normal circumstances to encounter shielded sources, i.e., fissile material embedded in a hydrogenous container intended to scatter and absorb neutrons. It is important to be able to recognize such configurations. We conducted exposures of the instrument to bare and shielded sources, with a much smaller exposure time than in Sections 4.1 and 4.4. The shielding was in the form of a $30 \mathrm{~cm} \oslash$ sphere of high density polyethylene (HDPE). Both neutron and $\gamma$-ray data were accumulated. Shown in Figure 10 are the neutron and $\gamma$ images of a bare ${ }^{252} \mathrm{Cf}$ source. The relatively strong $\gamma$ emission and the high sensitivity of NSPECT to $\gamma$ rays produces a better image.

Figure 11 shows the corresponding images of the same source, but inside the moderating HDPE sphere. The source was moved $0.2 \mathrm{~m}$ in the negative $\mathrm{x}$ direction in the laboratory to accommodate for the addition of the HDPE sphere. The reduced intensity of the neutron source is evident (for the same exposure length) in Figure 11a. and thus individual event circles can be seen. About fifty neutron events are registered, enough for a coherent neutron source identification and location. Little attenuation is seen in the $\gamma$ image (Figure 11b.) 


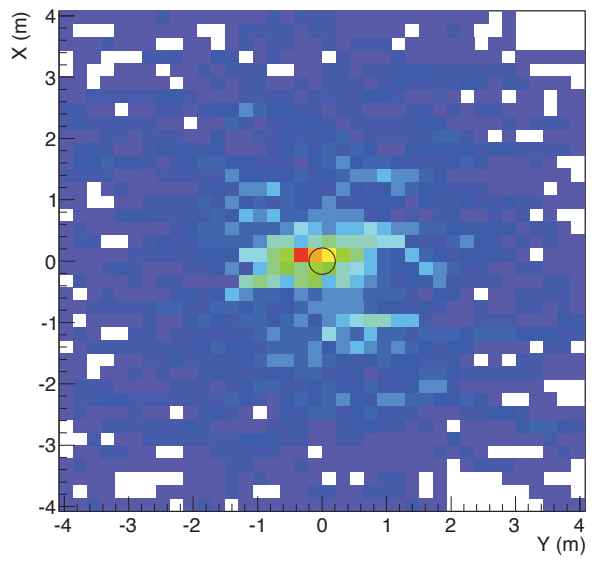

10a. The neutron image of a bare ${ }^{252} \mathrm{Cf}$ source.

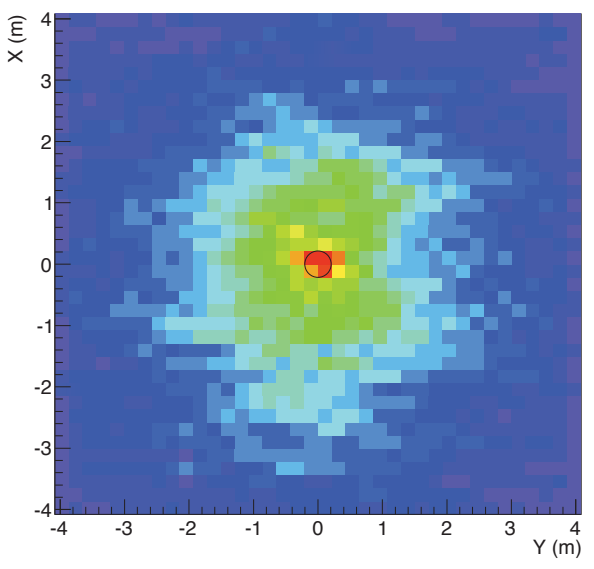

10b. The $\gamma$ image of a bare ${ }^{252} \mathrm{Cf}$ source.

Figure 10. Unmoderated neutron and gamma images for ${ }^{252} \mathrm{Cf}$.

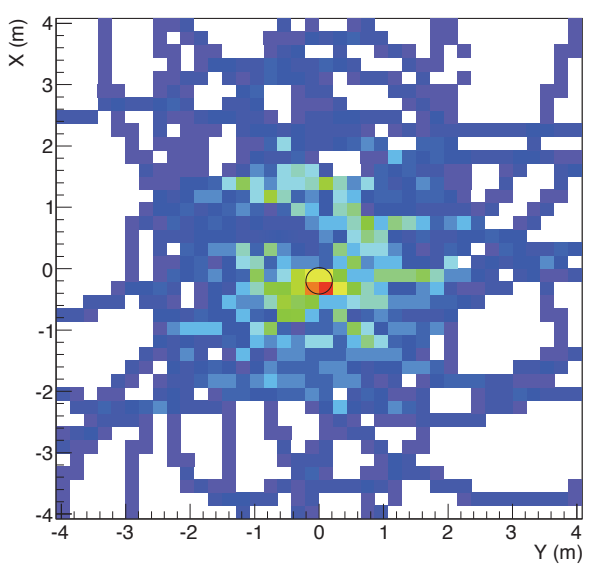

11a. The neutron image of a moderated ${ }^{252} \mathrm{Cf}$ source.

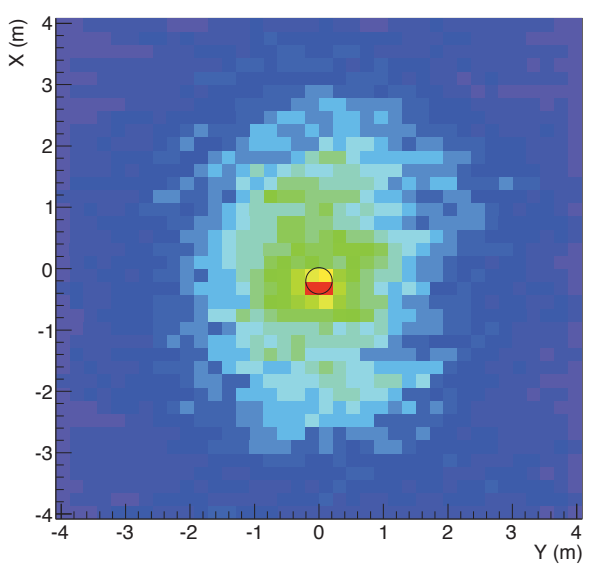

11b. The $\gamma$ image of a moderated ${ }^{252} \mathrm{Cf}$ source.

Figure 11. Moderated neutron and gamma images for ${ }^{252} \mathrm{Cf}$.

In Figures 12a. and b. are the neutron and $\gamma$ spectra, both moderated and unmoderated. The neutron signal is reduced considerably, but the source is still plainly present in the $\gamma$ signal. The basic exponential form of the Watt spectrum is still apparent, but greatly reduced in amplitude. No such significant modification of the spectrum or its intensity is seen in the $\gamma$ signal (Fig. 12b.) 


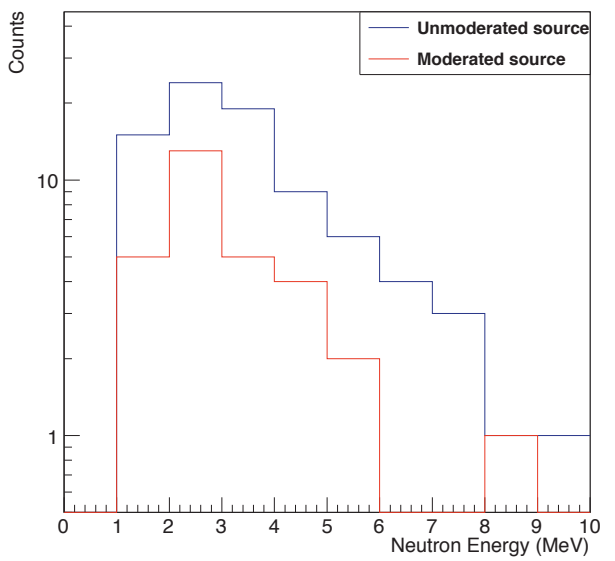

12a. The neutron spectrum from a moderated and unmoderated source.

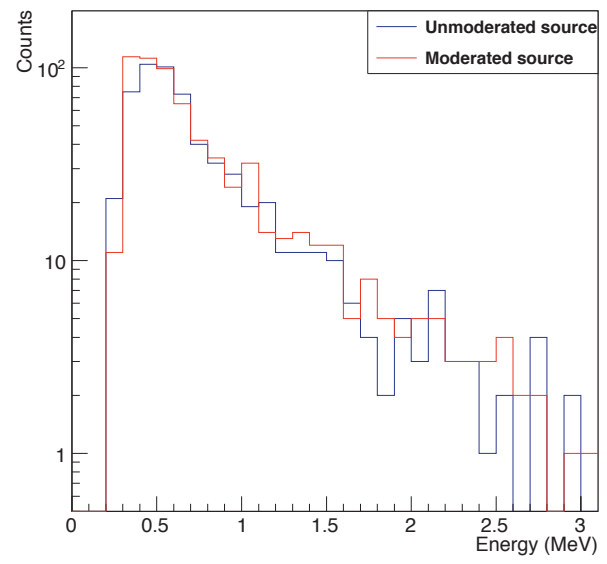

12b. The $\gamma$ spectrum from a moderated and unmoderated source.

Figure 12. Moderated and unmoderated spectra for a ${ }^{252} \mathrm{Cf}$ source.

\subsection{Special Nuclear Materials}

For this investigation we analyzed data from depleted uranium and enriched plutonium sources. The depleted uranium source saw a neutron rate that was four times the background neutron rate, while the $\gamma$ rate was six times the background $\gamma$ rate. The raw $\gamma$ image is shown in Figure 13a. and the neutron events are shown in Figure 13b.

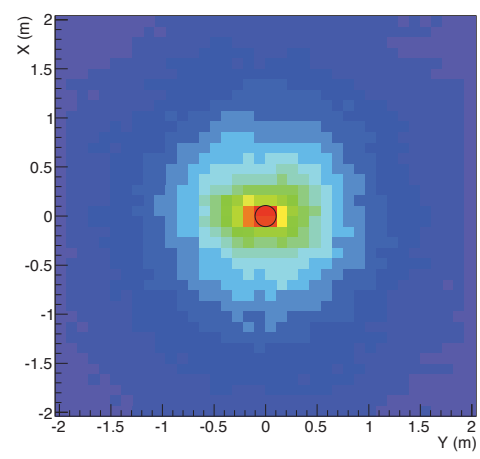

13a. The $\gamma$ image of depleted uranium.

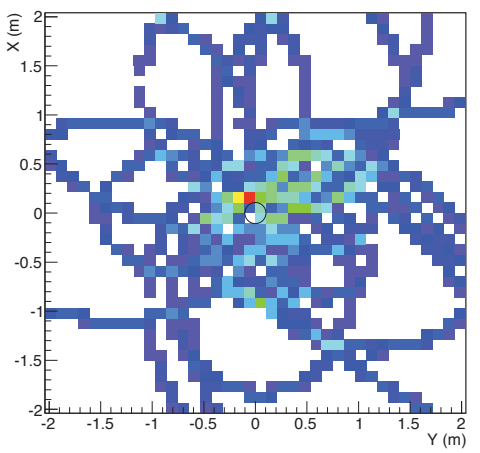

13b. The neutron image of depleted uranium.

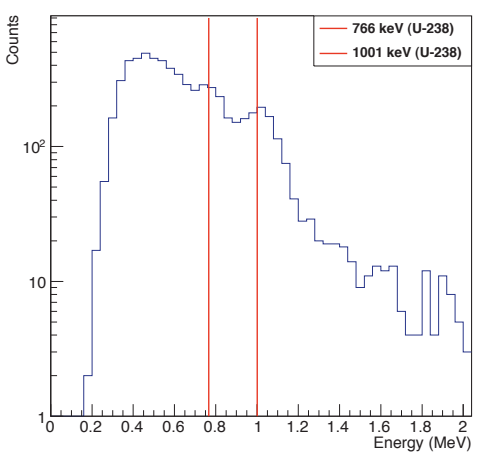

13c. Gamma count spectrum of depleted uranium showing the characteristic lines from ${ }^{238} \mathrm{U}$.

Figure 13. Images from a depleted uranium source.

Looking at the $\gamma$ spectrum for the depleted uranium source shows signatures from ${ }^{238} \mathrm{U}$ which has spectral lines at 0.766 and $1.00 \mathrm{MeV}$. The spectrum shown in Figure 13c. implements a $\pm 12^{\circ} \mathrm{ARM}$ cut to reduce the contributions of the background events to the spectral shape.

A similar analysis was performed with an enriched plutonium source (Figure 14). A sample of plutonium contains ${ }^{239} \mathrm{Pu}$ and ${ }^{240} \mathrm{Pu}$, which have spectral lines at 0.41 and $0.64 \mathrm{MeV}$ respectively. Here, the $\gamma$ rate was $\sim$ two times the $\gamma$ background, while the neutron rate was three times the neutron background. 
This analysis suggests that looking at the neutron and $\gamma$ events in tandem yields more information about the location and type of source than looking at either of the data sets alone.

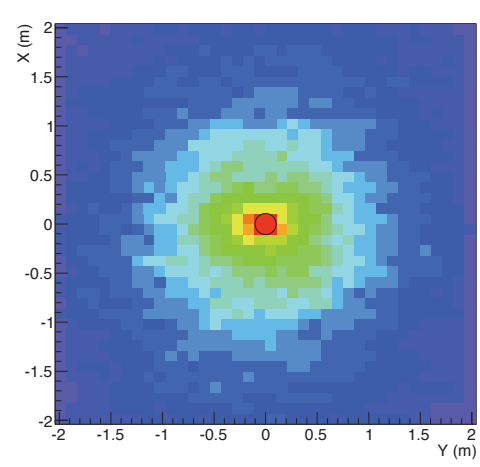

14a. The $\gamma$ image of plutonium.

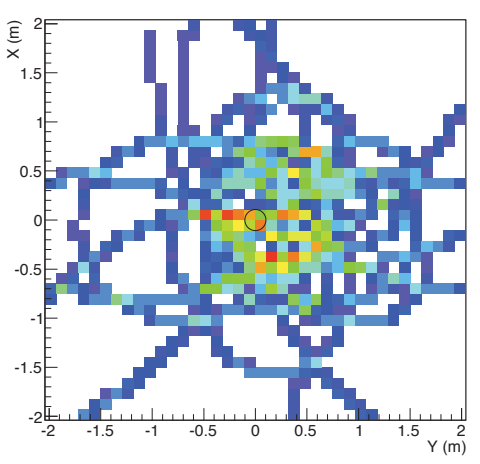

14b. The neutron image of plutonium.

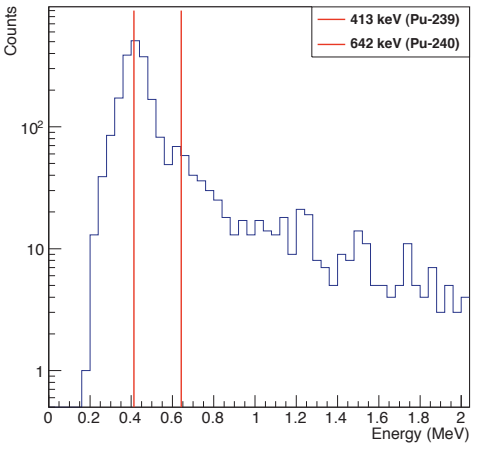

14c. Gamma count spectrum of plutonium showing the characteristic lines from ${ }^{239} \mathrm{Pu}$ and ${ }^{240} \mathrm{Pu}$.

Figure 14. Images from a plutonium source.

\subsection{Field Applications}

To examine the response of NSPECT in a field demonstration, we took the instrument out to a quonset hut located on the UNH campus in which we placed a ${ }^{137} \mathrm{Cs}$ source and a ${ }^{252} \mathrm{Cf}$ source in two separate locations. In order to determine a safe standoff distance for radiation workers, hand-held neutron and gamma radiation detectors are typically used to find a distance where counts above background were sufficiently low (a distance of $10 \mathrm{~m}$ from the quonset hut). We operated NSPECT from the back of an SUV at this location, positioned such that the quonset hut was completely within the field of view of the instrument. An integrated camera located on the front panel of NSPECT provided an image of the field of view, on which the source images were overlaid. The sources were not shielded, other than the moderation due to the quonset hut walls and the mass of air between the sources and the SUV. Even with this moderation, the neutron and gamma images were still clearly visible, as seen in Figure 16b.

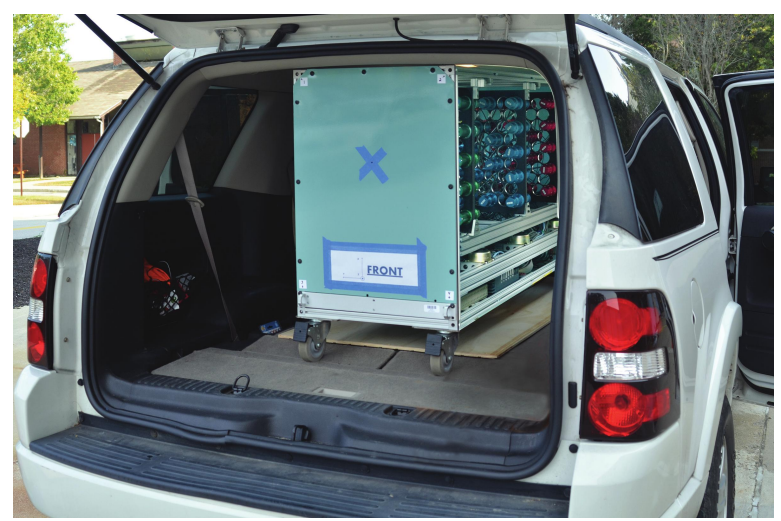

Figure 15. NSPECT loaded in the back of a standard size SUV. 


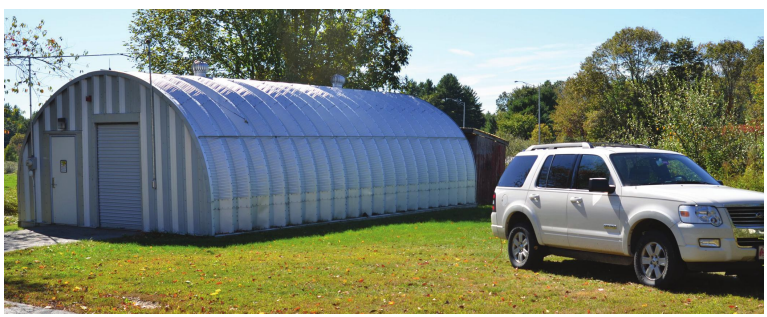

16a. NSPECT was loaded in an SUV and directed towards a quonset hut where a ${ }^{252} \mathrm{Cf}$ and ${ }^{137} \mathrm{Cs}$ source were located.

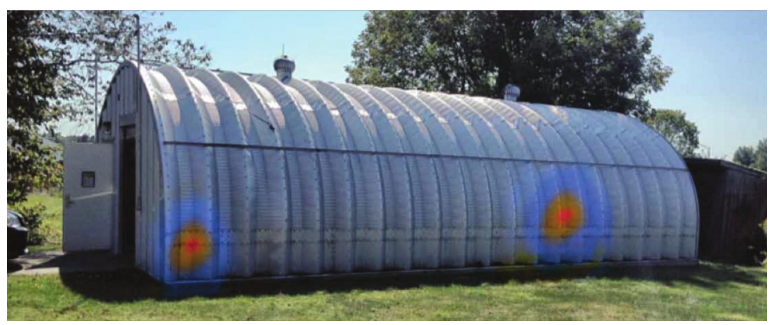

16b. The neutron detected image (left) and the gamma detected image (right) overlaid on an image of the field of view.

Figure 16. Field application of NSPECT exposed to sources hidden inside a quonset hut.

\section{CONCLUSIONS}

NSPECT, the neutron spectrometer telescope, a joint venture of Michigan Aerospace Corp. and the University of New Hampshire has been exercised as an operating imaging spectrometer. Its ability to simultaneously image and measure neutrons and $\gamma$ rays makes it a powerful tool for a variety of domestic forensic and remote sensing radiation situations. When fully populated with seventy-seven cells per plane, the instrument efficiency will increase by $9.5 \times$ for both neutrons and $\gamma$ rays.

\section{ACKNOWLEDGMENTS}

This work was and is supported by the Defense Threat Reduction Agency through Michigan Aerospace Corp., contracts HDTRA1-08-C-0077 and HDRTA1-11-C-0054. It builds on research started with funding from NNSA NA-22.

\section{REFERENCES}

[1] Schoenfelder, V. et al, "Instrument Description and Performance of the Imaging Gamma-Ray Telescope COMPTEL Aboard the Compton Gamma-Ray Observatory", Ap. J. 86, 657-692, (1993).

[2] Descalle, M., Labov, S. "Estimates of Neutron Reaction Rates in Three Portable He-3 Proportional Counters", LLNL, DE2009-964095, 10, (2007).

[3] Ryan, James M. et al., "An Imaging Neutron/Gamma-Ray Spectrometer", Proc. SPIE, 850905, (2012). 